\title{
A Multicentre, Randomized, Double-Blind, Placebo-Controlled, Crossover Study To Investigate the Efficacy, Safety, Tolerability, and Pharmacokinetics of Repeat Doses of Inhaled Nemiralisib in Adults with Persistent, Uncontrolled Asthma ${ }^{\$}$
}

\author{
Sanjeev Khindri, Anthony Cahn, Malcolm Begg, Mickael Montembault, Claudia Leemereise,
} Yi Cui, Annabel Hogg, Hannah Wajdner, Shuying Yang, Jon Robertson, J. Nicole Hamblin, Andrea Ludwig-Sengpiel, Oliver Kornmann, and Edith M. Hessel

\begin{abstract}
Respiratory Therapy Area Unit (S.K.), Clinical Pharmacology Science and Study Operations (M.M.), Global Clinical Safety and Pharmacovigilance (Y.C.), and Clinical Pharmacology Modelling and Simulation, Quantitative Sciences, RD Projects Clinical Platforms and Sciences (S.Y.), GSK, Stockley Park, Uxbridge, Middlesex, United Kingdom; Discovery Medicine (A.C.), Refractory Respiratory Inflammation Discovery Performance Unit (M.B., H.W., J.N.H., E.M.H.), Clinical Pharmacology Science and Study Operations (A.H.), and Clinical Statistics (J.R.), GSK, Stevenage, Hertfordshire, United Kingdom; Clinical Operations Department, GSK, Zeist, The Netherlands (C.L.); KLB Gesundheitsforschung Lübeck, Lübeck, Germany (A.L.-S.); and IKF Pneumologie Frankfurt, Clinical Research Centre Respiratory Diseases, Frankfurt, Germany (O.K.)
\end{abstract}

Received April 3, 2018; accepted September 11, 2018

\section{ABSTRACT}

Phosphoinositide 3-kinase $\delta(\mathrm{PI} 3 \mathrm{~K} \delta$ ) is a lipid kinase involved in leukocyte recruitment and activation. Activation of PI3K $\delta$ has been linked to airway inflammation and asthma pathogenesis. This randomized, double-blind, placebo-controlled, crossover study investigated the efficacy, safety, tolerability, and pharmacokinetics of a PI3K $\delta$ inhibitor, nemiralisib (GSK2269557), in patients with persistent, uncontrolled asthma. Patients $(n=50)$ received once-daily inhaled nemiralisib $(1000 \mu \mathrm{g})$ or placebo for 28 days, with a crossover to the alternative treatment following a 4-week washout period. Spirometry demonstrated no discernible difference in trough forced expiratory volume in 1 second $\left(\mathrm{FEV}_{1}\right)$ from baseline (adjusted posterior median $7 \mathrm{ml}$; 95\% credible interval $-83,102 \mathrm{ml}$ ) between nemiralisib and placebo treatment at day 28 (primary endpoint). These results were supported by most secondary endpoints, including weighted mean $\mathrm{FEV}_{1}$ ( $0-4$ hours) and change in trough forced vital capacity at day 28 . Nemiralisib was generally well-tolerated, with few side effects except for post-inhalation cough (nemiralisib: 35\%; placebo: 9\%). At day 14, sputum interleukin (IL)-5, IL-13, IL-6, and IL-8 levels were reduced by a median of $17 \%, 7 \%, 15 \%$, and $8 \%$, respectively, when comparing nemiralisib with placebo $[n=15$ (IL-5, IL-8) or 16 (IL-6, IL-13); posterior probability of a true ratio $>0 \%: 78 \%$, $64 \%, 76 \%$, and $63 \%$, respectively]. These results suggest that nemiralisib inhibited PI3K $\delta$ locally; however, this did not translate into meaningful clinical improvement. Further studies will investigate the potential efficacy of nemiralisib in patients with asthma with other specific more severe phenotypes, including those who are colonized with bacteria and frequently exacerbate.
This work has not been previously presented.

S.K., A.C., M.B., M.M., Y.C., A.H., H.W., S.Y., J.R., and E.M.H. are employees of, and hold stocks/share options in, GSK. C.L. is an employee of GSK. J.N.H. was an employee of GSK at the time this study was conducted and is a stockholder in GSK. J.N.H. and E.M.H. are listed as coinventors on patents relating to nemiralisib. A.L.-S. and O.K.'s institutions each received honoraria for conducting the clinical trial described in this paper.

This work was supported by GSK (ClinicalTrials.gov Identifier: NCT02567708). https://doi.org/10.1124/jpet.118.249516.

S This article has supplemental material available at jpet.aspetjournals.org.

\section{Introduction}

Asthma, a chronic respiratory disease, affects approximately 358 million people worldwide, and the prevalence is increasing, rising by $12.6 \%$ globally between 1990 and 2015 (Soriano et al., 2017). Asthma is ranked 23rd among the global burden of disease causes, contributing $1.1 \%$ of global disability-adjusted life years (Soriano et al., 2017). Common symptoms of asthma include shortness of breath, chest tightness, wheeze, and cough, and patients may experience periodic flare-ups (exacerbations) (www.ginasthma.org).

ABBREVIATIONS: ACT, asthma control test; AE, adverse event; COPD, chronic obstructive pulmonary disease; Crl, credible interval; ECG, electrocardiogram; FeNO, fractional exhaled nitric oxide; FEV ${ }_{1}$, forced expiratory volume in 1 second; FVC, forced vital capacity; ICS, inhaled corticosteroids; IL, interleukin; ITT, intent-to-treat; LABA, long-acting $\beta_{2}$-agonist; LLQ, lower limit of quantification; PD, pharmacodynamics; PEF, peak expiratory flow; PI3K, phosphoinositide 3-kinase; PK, pharmacokinetics; PP, per-protocol; QC, quality control; SHIP1, Src homology 2-containing inositol-5'-phosphatase 1 ; TNF, tumor necrosis factor. 
Current guidelines recommend inhaled corticosteroids (ICS) for the initial treatment of asthma (www.ginasthma.org); however, a subpopulation of patients with asthma remains uncontrolled despite taking ICS, and long-term ICS usage may be associated with side effects (Lipworth, 1999; Reddy and Little, 2013). Alternative therapies include leukotriene receptor antagonists and, in patients experiencing persistent symptoms or exacerbations, long-acting $\beta_{2}$-agonist (LABA) in free- or fixed-dose combination with ICS (www.ginasthma. org). Patients with severe asthma or persistent symptoms despite these treatments may be prescribed biologics, such as anti-IgE or anti-interleukin (IL)-5 agents (www.ginasthma. org). However, whereas biologics may be beneficial in reducing exacerbations, they are costly and they may not be cost effective for many patients (Wu et al., 2007; Menzella et al., 2016). Thus, there remains a need for novel therapies to achieve disease control in patients with asthma.

Asthma is characterized by chronic airway inflammation and hyper-responsiveness to inhaled allergens and indirect stimuli (www.ginasthma.org). In atopic individuals, $\mathrm{T}$ cells produce inflammatory mediators upon antigen exposure, such as IL-4, IL-5, and IL-13, which recruit and activate mast cells, eosinophils, and IgE-producing B cells, mediating local allergic inflammation and contributing to the pathogenesis of asthma (Robinson et al., 1992, 1993; Matangkasombut et al., 2009; Deo et al., 2010). Correspondingly, an increase in inflammatory cytokines, such as IL-6 and IL-8, is observed in the lungs of patients with asthma experiencing an exacerbation (Yokoyama et al., 1995; Norzila et al., 2000; Maneechotesuwan et al., 2007).

Phosphoinositide 3-kinases (PI3Ks) are a large family of lipid-signaling kinases (Park et al., 2008). The class I family of PI3Ks phosphorylates the membrane phospholipid phosphatidylinositol 4,5-biphosphate into phosphatidylinositol 3,4,5-trisphosphate, which acts in various signaling pathways (Vanhaesebroeck et al., 2010). The class IA PI3K $\delta$ isoform is expressed predominantly in leukocytes (Chantry et al., 1997) and has roles in mediating antigen receptor and cytokine signaling in $\mathrm{T}$ cells (including Th2 cells), mast cells, and B cells, as well as in eosinophil migration (Clayton et al., 2002; Okkenhaug et al., 2002, 2007; Ali et al., 2004; Patton et al., 2006; Nashed et al., 2007; Stark et al., 2015; Way et al., 2016). In asthma mouse models, PI3K $\delta$ inhibition has been shown to attenuate recruitment of inflammatory cells, including lymphocytes and eosinophils, and reduce the release of proinflammatory T cell cytokines (Lee et al., 2006). Additionally, $\mathrm{PI} 3 \mathrm{~K} \delta$ inhibition may prevent $\mathrm{IgE}$ release from $\mathrm{B}$ cells and reduce degranulation of mast cells (Ali et al., 2004; Lee et al., 2006).

Src homology 2-containing inositol-5'-phosphatase 1 (SHIP1) is predominantly expressed in hematopoietic cells and dephosphorylates phosphatidylinositol 3,4,5-trisphosphate to phosphatidylinositol 4,5-biphosphate, thereby inducing selective downregulation of the PI3K $\delta$ pathway (Leaker et al., 2014). In patients with mild-to-moderate asthma, activation of SHIP1 with oral AQX-1125 was shown to significantly attenuate allergen-induced responses, accompanied by a trend toward reduced recruitment of eosinophils, neutrophils, and macrophages (Leaker et al., 2014).

Nemiralisib (GSK2269557) is a potent and highly selective inhaled $\mathrm{PI} 3 \mathrm{~K} \delta$ inhibitor being developed as a potential therapeutic for the treatment of inflammatory airway diseases (Down et al., 2015; Cahn et al., 2017). The effects of nemiralisib $(1000 \mu \mathrm{g})$ in patients with stable chronic obstructive pulmonary disease (COPD) were recently investigated in a randomized, double-blind, placebo-controlled study, which found a reduction in sputum IL-6 (29\%) and IL-8 (32\%) levels following inhalation of nemiralisib, suggesting nemiralisib may reduce airway inflammation (Cahn et al., 2017), consistent with reduced disease severity (Franciosi et al., 2006; Hacievliyagil et al., 2006). The present proof-of-concept study was the first administration of inhaled nemiralisib (1000 $\mu \mathrm{g}$ once daily for 28 days) to patients with persistent, uncontrolled asthma who had not received ICS or LABA treatment of $\geq 12$ weeks prior to the first dose of study medication. We aimed to investigate the efficacy, safety, tolerability, and systemic pharmacokinetics $(\mathrm{PK})$ of nemiralisib in this patient group.

\section{Materials and Methods}

Study Design. This was a multicentre, randomized, double-blind, placebo-controlled, two-period crossover study in patients with persistent, uncontrolled asthma, currently not treated with an ICS or a LABA. The study was conducted at 12 centers in Germany, of which three closed without activity and one center experienced a screening failure. Data were collected from eight centers between October 7, 2015 and September 28, 2016 (ClinicalTrials.gov identifier: NCT02567708).

Patients were screened for eligibility up to 28 days before randomization. Those patients meeting the eligibility criteria entered a run-in period of approximately 2 weeks, during which baseline asthma status was measured and safety evaluations were performed. At the end of the run-in period, eligible patients were randomized to one of two sequences. Patients randomized to sequence one received matched placebo once daily for $28 \pm 2$ days in an initial treatment period (treatment period 1) and nemiralisib $1000 \mu \mathrm{g}$ once daily for $28 \pm 2$ days in a second treatment period (treatment period 2). Patients randomized to sequence two received nemiralisib $1000 \mu \mathrm{g}$ once daily for $28 \pm 2$ days in treatment period 1 and matched placebo once daily for $28 \pm 2$ days in treatment period 2 . There was a washout period of $\geq 4$ weeks between treatment periods. Patients visited the clinic on the first day of treatment (day 1), and at days 7, 14, and 28 of each treatment period, and attended a follow-up visit 1 to 2 weeks after their last dose.

Nemiralisib and placebo were administered using the DISKUS dry powder inhaler (owned by or licensed to the GSK group of companies). The dose of nemiralisib was selected based on previous safety, tolerability, and biomarker data in patients with COPD; the same nemiralisib formulation and device were also used (Cahn et al., 2017). Use of this dose is also supported by extensive preclinical research (Down et al., 2015).

To maintain blinding, the devices used to administer nemiralisib and placebo were identical in appearance. An interactive web response system was used to assign patients to a treatment sequence, and participating sites were provided with only the number of the device to use, not the treatment sequence.

The study was conducted in accordance with the International Conference on Harmonization Good Clinical Practice and the Declaration of Helsinki. All patients provided written informed consent prior to participation in the study.

Patients. Patients were eligible for inclusion if they were aged 18-70 years with persistent, uncontrolled bronchial asthma treated only with an intermittent short-acting $\beta_{2}$-agonist or other noncorticosteroid controllers, and had not received ICS or LABA treatment of $\geq 12$ weeks prior to the first dose of study medication. In addition, patients were required to have a best prebronchodilator forced expiratory volume in 1 second $\left(\mathrm{FEV}_{1}\right) \geq 60 \%$ of the predicted normal value at screening, and a $\mathrm{FEV}_{1}$ increase by $\geq 12 \%$ and $\geq 200 \mathrm{ml}$ over the baseline value within 
$10-40$ minutes of inhalation of $400 \mu \mathrm{g}$ salbutamol via a metered dose inhaler.

Key exclusion criteria included a history of life-threatening asthma, severe asthma exacerbation, or respiratory infection; and current smokers or patients with a history of smoking within 6 months of screening. Full exclusion and withdrawal criteria are listed in Supplemental Materials.

All patients were allowed to use salbutamol on an as-needed basis during the entire study, except for the 4 hours prior to each $\mathrm{FEV}_{1}$ and fractional exhaled nitric oxide (FeNO) test conducted at the clinic, if possible.

Study Objectives and Endpoints. The primary study objective was to investigate the efficacy of inhaled nemiralisib administered once daily for 28 days in patients with persistent, uncontrolled asthma, compared with placebo. The primary endpoint was defined as the change from baseline in trough $\mathrm{FEV}_{1}$ at day 28. This primary endpoint chosen as $\mathrm{FEV}_{1}$ is a recognized efficacy measure in this population and enables direct comparison with established therapeutics.

The secondary study objectives were to characterize the clinical response, safety, and tolerability, and plasma PK of inhaled nemiralisib administered once daily for 28 days in patients with persistent, uncontrolled asthma. Secondary efficacy endpoints were as follows: change from baseline in trough $\mathrm{FEV}_{1}$ at days 7 and 14; percent change from baseline in trough $\mathrm{FEV}_{1}$ at days 7, 14, and 28; weighted mean (0-4 hours) $\mathrm{FEV}_{1}$ at day 28; change from baseline in forced vital capacity (FVC) at days 7, 14, and 28; change from baseline in $\mathrm{FEV}_{1} / \mathrm{FVC}$ at days 7, 14, and 28; change from baseline in daily $\mathrm{FEV}_{1}$ (morning) and peak expiratory flow (PEF) (morning and evening) averaged over the treatment period; change from baseline in asthma control test (ACT) score at day 28; change from baseline in trough $\mathrm{FeNO}$ at days 7, 14, and 28; and the mean number of inhalations per day of rescue medication. Safety endpoints included the incidence of adverse events (AEs), monitoring of vital signs, 12-lead electrocardiogram (ECG), and clinical laboratory tests (hematology and clinical chemistry). PK assessments to determine the trough plasma concentration after 7, 14, and 28 days of treatment and day 28 plasma exposure up to 3.5 hours postdose were also conducted. Exploratory endpoints included pharmacodynamic (PD) analyses, which investigated the levels of proinflammatory cytokines in induced sputum prior to and after 14 days of treatment.

Efficacy Assessments. FEV 1 and FVC were measured in the clinic using a spirometer in accordance with the American Thoracic Society/European Respiratory Society standards (Miller et al., 2005). Single time point spirometry was performed predose on days 1,7 , and 14, and serial spirometry was performed on day 28: predose, and 1-4 hour postdose of each treatment period. Daily $\mathrm{FEV}_{1}$ and PEF were measured morning and evening by patients using a handheld device before any rescue salbutamol use. FeNO was measured at days 1, 7, 14 , and 28 of each treatment period, using a handheld electronic device in accordance with the American Thoracic Society/European Respiratory Society Recommendations (American Thoracic Society and European Respiratory Society, 2005), and interpreted as per the American Thoracic Society Clinical Practice Guideline (Dweik et al., 2011). Patients self-completed the five-item ACT on days 1 and 28 before any other assessment at a clinic visit.

Safety Assessments. AE monitoring was conducted throughout the study. In each treatment period, 12-lead ECG was performed at screening and predose on days 1,7 , and 28 . Vital signs were measured at screening; predose on days 1, 7, 14, and 28; and at the follow-up visit. Routine laboratory assessments were carried out at screening and predose on days 1,14 , and 28 .

PK Assessments. Blood samples were collected via an indwelling cannula or by direct venipuncture predose on days 7,14 , and 28 . In addition, on day 28 , samples were taken between 5 and 10 minutes postdose and also between 2.5 and 3.5 hours postdose. Plasma samples were analyzed for nemiralisib using an internally validated analytical method based on protein precipitation, followed by high performance liquid chromatography/tandem mass spectrometry analysis. The lower limit of quantification (LLQ) was $20 \mathrm{pg} / \mathrm{ml}$ using a $50 \mu \mathrm{l}$ aliquot of ethylenediaminetetraacetic acid plasma. The higher limit of quantification was $10,000 \mathrm{pg} / \mathrm{ml}$.

Calibration standards and quality control (QC) samples prepared at three different analyte concentrations were analyzed with each batch of samples. For the analysis to be acceptable, no more than one-third of the $\mathrm{QC}$ results were to deviate from the nominal concentration by more than $15 \%$, and at least $50 \%$ of the results from each QC concentration needed to be within $15 \%$ of nominal concentration. The applicable analytical runs met all predefined run acceptance criteria.

Cytokine Levels in Induced Sputum. Hypertonic salineinduced sputum samples were collected during screening (for confirmation of eligibility) and predose on days 1 and 14 in a subset of patients. Time points were selected to match those used previously in the study of nemiralisib in patients with COPD to enable comparison (Cahn et al., 2017). Cytokines [IL-4, IL-5, IL-6, IL-8, IL-13, and tumor necrosis factor (TNF)- $\alpha$ ] in sputum supernatant were analyzed using custom multiplex assay (V-plex) platform from Meso Scale Discovery (Gaithersburg, MD), according to the manufacturer's instructions. Imputed values (half of the LLQ) were used for cases in which the data obtained from a sample were below the LLQ.

Statistical Methods. A crossover design was selected to provide tighter precision for the treatment comparisons, compared with a parallel group design using the same number of patients. The sample size was determined by feasibility; however, this was deemed to provide sufficient precision around the estimation for the comparisons of interest to enable progression given positive data. Assuming the within-patient S.D. was similar to that observed in previous studies, it was estimated that the lower and upper bounds of the $95 \%$ credible intervals for the difference between nemiralisib and placebo for the primary endpoint with the proposed sample size would be within $\pm 94 \mathrm{ml}$ of the observed treatment difference. Approximately 50 patients were planned to be randomized to the study to ensure completion of both treatment periods by approximately 40 patients, assuming a $20 \%$ dropout rate. Randomization was stratified by patients who were able and willing to provide induced sputum samples (able to produce $>100 \mathrm{mg}$ induced sputum at screening or during the run-in period) and those who were not. Approximately 16 patients were planned to be randomized into the induced sputum stratum. Randomization to treatment sequence was in a 1:1 ratio into each stratum.

The primary efficacy analyses were based on the intent-to-treat (ITT) population (in which patients were analyzed according to the treatment they received), and a sensitivity analysis was performed in the per-protocol (PP) population, which included all patients with no major protocol violation. The secondary efficacy, safety, and PK analyses were conducted in the ITT, safety, and PK populations, respectively. Exploratory $\mathrm{PD}$ endpoints were assessed in the $\mathrm{PD}$ population. The safety population comprised all randomized patients, and the PK and PD populations comprised all patients in the ITT population who had a blood sample for PK analysis, or who participated in the sputum substudy, respectively.

Adjusted posterior medians and corresponding 95\% credible intervals (CrI) for the change from baseline in trough $\mathrm{FEV}_{1}$ were produced for both study groups, as well as the difference between nemiralisib and placebo. Posterior probabilities that the true difference was greater than various thresholds $(0,0.05$, and $0.1 \mathrm{l})$ were also produced. The change from baseline in FVC was analyzed in the same way.

The weighted mean (0-4 hours) $\mathrm{FEV}_{1}$ was calculated for each treatment period based on clinic $\mathrm{FEV}_{1}$ data obtained at predose and 1-4 hours postdose on day 28 . The weighted mean was derived by calculating the area under the curve, and then dividing by the actual relevant time interval.

Multiple readings $(\leq 8)$ were taken for each FeNO assessment, which were log-transformed and analyzed using the same technique as for the $\mathrm{FEV}_{1}$ analyses. Results were back-transformed to provide estimated geometric medians per treatment.

The ACT questionnaire had five possible response options for each question, with a score of 1 (poor control) to 5 (good control), and the scores from each question were summed to give a total score. 
Each efficacy endpoint was analyzed used a Bayesian model using a noninformative prior for all model parameters, and adjusting for the following covariates: patient-level baseline, adjusted period-specific baseline, period, and treatment. For each $\mathrm{FEV}_{1}$ outcome, including weighted mean (0-4 hours) $\mathrm{FEV}_{1}$, the baseline was the trough $\mathrm{FEV}_{1}$.

The plasma nemiralisib PK concentration-time data were summarized by study day and sampling time. The trough value of plasma nemiralisib concentration was determined as the concentration measured at predose on each study visit.

\section{Results}

Patient Population. Of 108 patients screened, 50 patients were eligible to enter the study, all of whom were randomized (24 patients received placebo, and 26 patients received nemiralisib during treatment period 1) (Fig. 1). The study was completed by $42(84 \%)$ patients. All patients were included in the safety population. Forty-four patients were included in the ITT population; six patients had an inclusion criteria deviation and were randomized when screening spirometry overread was not acceptable. Because they entered the study without spirometric evidence of asthma, they were excluded from the ITT population. Forty-two patients were included in the PK population and 41 in the PP population.
Sixteen patients (eight assigned to each treatment sequence) participated in the sputum substudy (PD population).

All patients were white, 22-67 years of age, and $56 \%$ were female (Table 1). Prestudy spirometry, FeNO (predose day 1 , treatment period 1), and blood eosinophil counts for the ITT population are shown in Table 2. The baseline characteristics of the patients who participated in the sputum substudy are provided in Supplemental Table 1.

Spirometry Assessments. Overall, posterior median trough $\mathrm{FEV}_{1}$ at day 28 increased from baseline in both nemiralisib and placebo treatment groups (Fig. 2). The adjusted median increase from baseline in $\mathrm{FEV}_{1}$ at day 28 (primary endpoint) was $27 \mathrm{ml}(95 \% \mathrm{CrI}:-66,117 \mathrm{ml})$ in the placebo group and $35 \mathrm{ml}(95 \% \mathrm{CrI}:-61,124 \mathrm{ml})$ in the nemiralisib treatment group. The posterior median treatment difference (nemiralisib vs. placebo) at day 28 was $7 \mathrm{ml}(95 \%$ CrI: $-83,102 \mathrm{ml}$ ). The posterior probability that the true difference between treatments is greater than zero at day 28 was 0.57 . This result indicates that patients in the nemiralisib group received no discernible benefit of treatment with respect to trough $\mathrm{FEV}_{1}$ at day 28 .

Similar results were observed in trough $\mathrm{FEV}_{1}$ at days 7 and 14; the posterior median trough $\mathrm{FEV}_{1}$ levels increased from baseline at days 7 and 14 in both nemiralisib and placebo

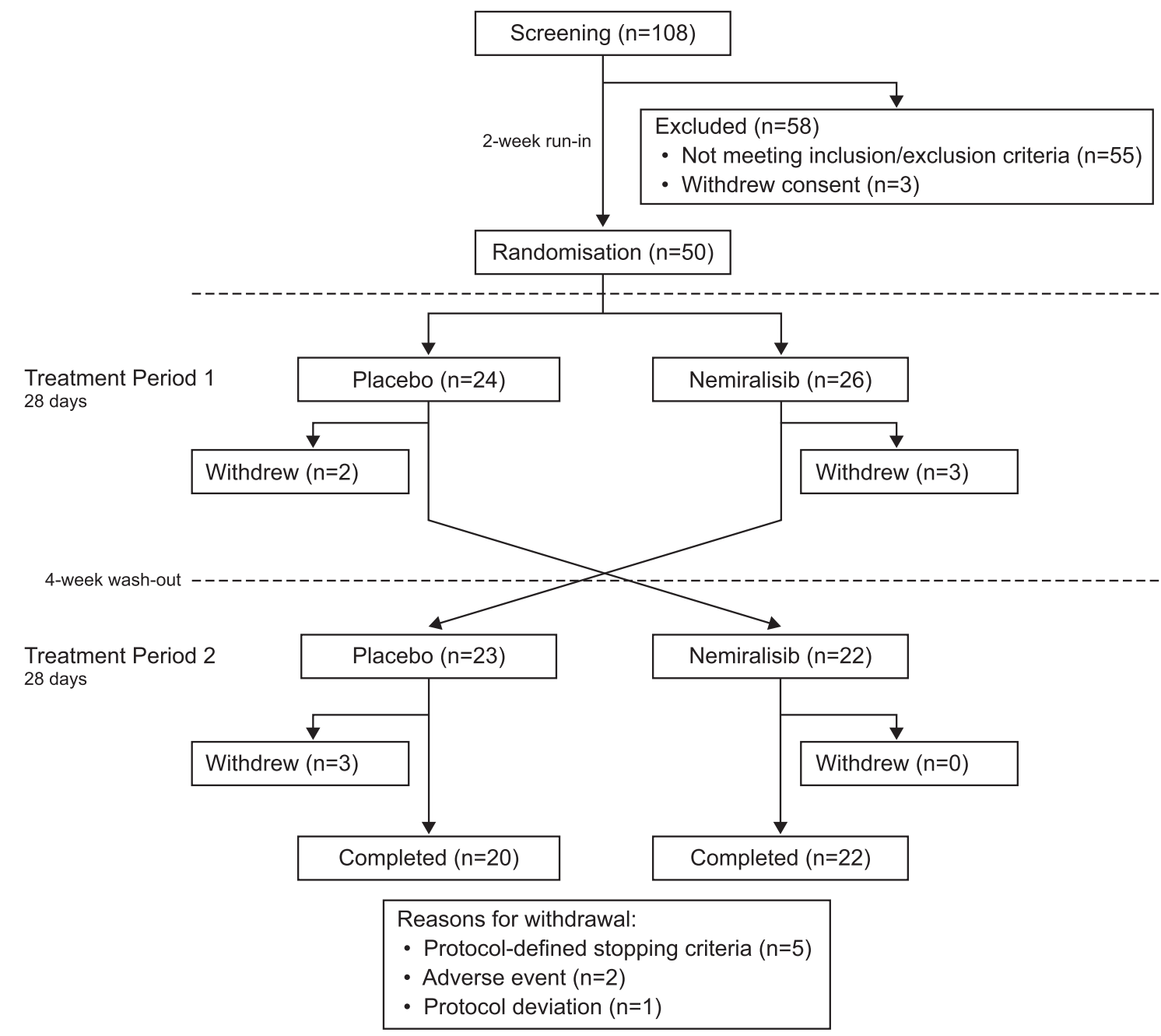

Fig. 1. CONSORT flow diagram of study participants. 
TABLE 1

Patient demographics (safety population)

\begin{tabular}{lc}
\hline \multicolumn{1}{c}{ Characteristic } & Total $(N=50)$ \\
\hline Age, years, mean (S.D.) & $44.5(13.86)$ \\
Sex & $28(56)$ \\
Female, $n(\%)$ & $22(44)$ \\
Male, $n(\%)$ & $26.94(3.66)$ \\
BMI, kg/m ${ }^{2}$, mean (S.D.)
\end{tabular}

BMI, body mass index.

treatment groups (Fig. 2). The posterior median treatment difference (nemiralisib vs. placebo) was $-9 \mathrm{ml}$ (95\% CrI: -151 , $131 \mathrm{ml})$ at day 7 , and $24 \mathrm{ml}(95 \% \mathrm{CrI}:-87,137 \mathrm{ml})$ at day 14 . The posterior probability that the true difference is greater than zero was 0.44 and 0.66 at days 7 and 14 , respectively. Sensitivity analyses in the PP population showed similar results to the ITT population.

Similarly, the adjusted median percent change from baseline in trough $\mathrm{FEV}_{1}$ showed a small increase in both groups at days 7,14 , and 28 , with the exception of day 7 in the nemiralisib group, in which a small percentage decrease was observed (Table 3). Overall, however, these data supported the observation that no discernible benefit was detected in patients treated with nemiralisib when compared with placebo-treated patients.

Values for the adjusted median of the weighted mean (0-4 hours) $\mathrm{FEV}_{1}$ at day 28, median trough $\mathrm{FVC}$, and the adjusted median change from baseline in trough $\mathrm{FEV}_{1} / \mathrm{FVC}$ for days 7,14 , and 28 are reported in Table 3 for both placebo and nemiralisib groups. Whereas small differences were observed, overall the data indicate that there was no benefit of nemiralisib treatment when compared with placebo. An increase from baseline was observed in daily evening $\mathrm{FEV}_{1}$ and $\mathrm{PEF}$ compared with daily morning $\mathrm{FEV}_{1}$ and $\mathrm{PEF}$, respectively, in both placebo and nemiralisib groups (Supplemental Table 2).

ACT. The total ACT score at day 28 increased from baseline by a posterior median of 1.4 points $(95 \% \mathrm{CrI}: 0.5,2.2)$ in the placebo group and 1.9 points (95\% CrI: $1.0,2.7)$ in the nemiralisib group (Table 3 ). The posterior median treatment difference at day 28 was 0.5 points $(95 \% \mathrm{CrI}:-0.6,1.7)$. The posterior probability that the true difference is greater than zero at day 28 was 0.81 .

FeNO. Posterior median change from baseline in trough FeNO is shown in Table 3. In the placebo group, trough FeNO levels increased from baseline by $3 \%$ at days 7 and 28, and marginally changed at day $14(-1 \%)$. In the nemiralisib group, trough FeNO decreased from baseline by $9 \%$ at day 7 and $5 \%$ at day 14 , and increased at day 28 by $3 \%$. The posterior median ratios (nemiralisib:placebo) at days 7 and 14 were $0.89(95 \%$

\section{TABLE 2}

Patient baseline characteristics measured at screening (ITT population)

These data were obtained by post hoc analyses.

\begin{tabular}{lc}
\multicolumn{1}{c}{ Characteristic } & Total $(N=44)$ \\
\hline Predicted normal FEV $_{1}, \%$, mean (range) & $73.87(60.3,96.5)$ \\
$\mathrm{FEV}_{1} / \mathrm{FVC}, \%$, mean (range) & $67.98(45.9,87.2)$ \\
$\mathrm{FEV}_{1}$ reversibility, $\%$, mean (range) & $21.17(11.9,50.8)$ \\
$\mathrm{FeNO}, \mathrm{ppb}$, geo mean & 28.64 \\
Blood eosinophil count, $10^{9} /$, geo mean & 0.266 \\
\hline
\end{tabular}

geo, geometric; ppb, parts per billion.

${ }^{a}$ Measurements taken at pre-dose day 1 of treatment period 1.

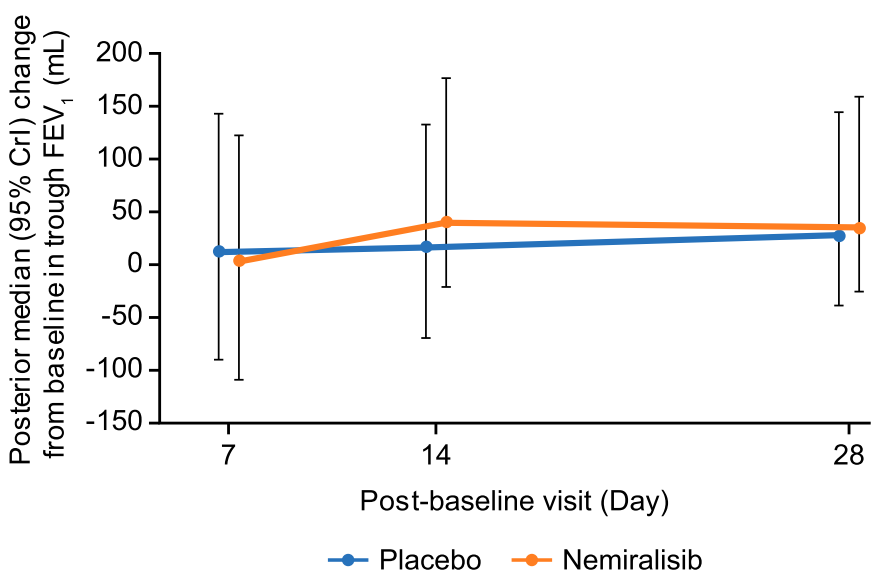

Fig. 2. Adjusted change from baseline in trough $\mathrm{FEV}_{1}$ (L) by visit (ITT population).

CrI: $0.78,1.00)$ and 0.95 (95\% CrI: 0.83, 1.09), respectively, favoring treatment with nemiralisib compared with placebo. The posterior median ratio at day 28 was 1.00 (95\% CrI: 0.84, 1.18). The posterior probability that the true treatment ratio is less than one was $0.97,0.76$, and 0.51 at days 7,14 , and 28 , respectively.

Use of Rescue Medication. The number of inhalations per day of rescue medication [mean (S.D.)] was lower in the nemiralisib group [2.7 (1.53)] than the placebo group [3.0 (1.86)]. The percentage of rescue-free days [mean (S.D.)] was marginally higher in the placebo group [49.5 (35.04)] compared with the nemiralisib group [47.3 (34.79)].

PK. With the exception of three patients, all nemiralisibtreated patients had a quantifiable plasma concentration of nemiralisib 5-10 minutes following inhalation, suggesting quick absorption of the drug. The plasma concentration of nemiralisib was similar, 5-10 minutes and 2.5-3.5 hours after dosing (Supplemental Table 3), suggesting a slow decline in plasma concentration. For the majority of patients, trough plasma concentration was stable after day 7. Mean trough levels of nemiralisib (predose values) were 556, 521, and $649 \mathrm{pg} / \mathrm{ml}$ on days 7, 14, and 28, respectively (Supplemental Table 3). The overlapping confidence intervals suggest there was no significant change in trough concentration between these time points.

Safety. A total of 31/50 (62\%) patients experienced AEs, including 25/48 (52\%), who reported AEs while receiving nemiralisib and $15 / 47$ (32\%) while receiving placebo (Table 4). AEs considered by the investigators to be drugrelated occurred at a higher frequency in the nemiralisib group (35\%) compared with the placebo group (4\%). Two (4\%) patients in the nemiralisib group discontinued treatment due to AEs: one due to acute bronchitis and another to exertional dyspnoea; neither was considered by the investigator to be treatment-related.

The most commonly reported AE was post-inhalation cough, with more patients reporting cough in the nemiralisib group $(17 / 48,35 \%)$ compared with placebo $(4 / 47,9 \%) ; 14$ patients in the nemiralisib group and two patients in the placebo group experienced a cough considered by the investigator to be treatment-related. Cough commonly occurred immediately after inhalation of nemiralisib and was generally mild or moderate in intensity and short of duration, with no patient discontinuing treatment due to cough. 
TABLE 3

Summary of statistical analysis of secondary endpoint data (ITT population)

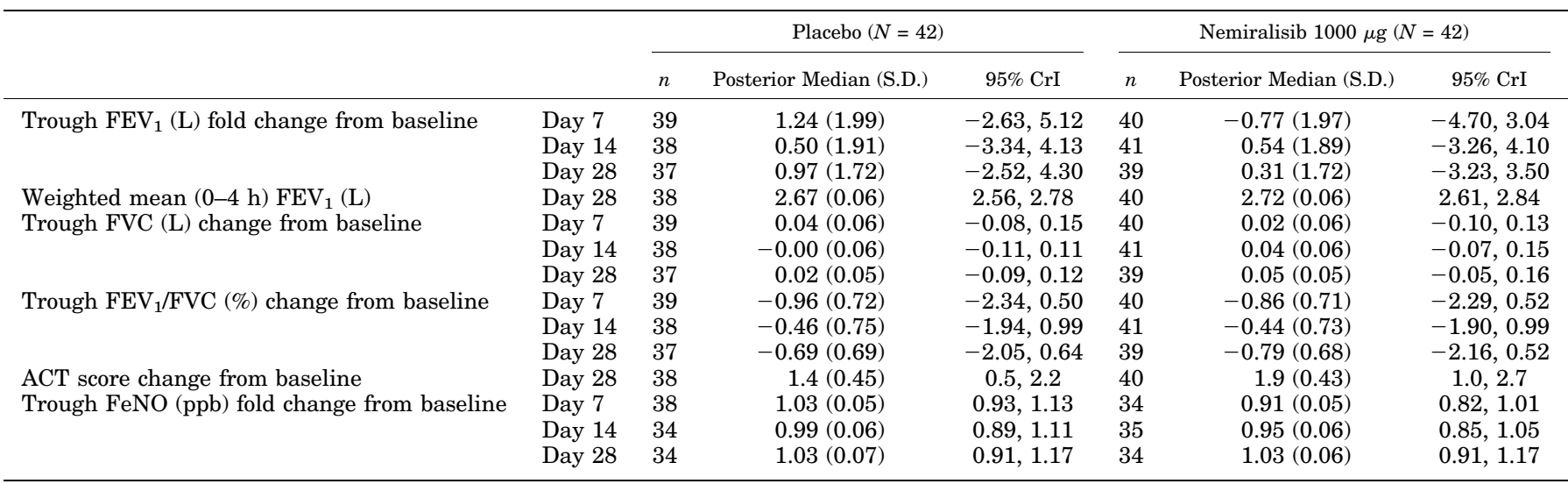

ppb, parts per billion

There was no increased report of AEs due to infections/ infestations in patients receiving nemiralisib compared with placebo $(3 / 48,6 \%$ vs. $8 / 47,17 \%)$, and the majority of infection/infestation-related AEs were nasopharyngitis. No serious AEs or deaths were reported, and there were no trends or changes of clinical concern in laboratory evaluations, vital signs, or ECG for nemiralisib when compared with placebo.

Cytokine Levels in Induced Sputum. Levels of inflammatory cytokines IL-5, IL-6, IL-8, IL-13, and TNF- $\alpha$ were measured in induced sputum samples, and the adjusted posterior median change from baseline for each cytokine is shown in Table 5. When comparing cytokine levels across treatment groups, a 17\% reduction in IL-5 levels in nemiralisib-treated patients was observed (posterior probability that the true ratio is less than one was 0.78 ). The ratio between nemiralisib and placebo treatment was 0.93 (reduction of 7\%) for changes in IL-13 (posterior probability that the true ratio is less than one was 0.64 ). The ratio between nemiralisib and placebo treatment of IL- 6 and IL-8 levels was 0.85 (a reduction of $15 \%$ ) and 0.92 (a reduction of $8 \%$ ), respectively (posterior probability that the true ratio is less than one was 0.76 for IL-6, and 0.63 for IL-8). No notable changes were observed in sputum TNF- $\alpha$ levels. Measurement of IL-4 was also attempted, but all values were below the assay LLQ.

TABLE 4

Summary of on-treatment adverse events (safety population)

\begin{tabular}{lccc}
\hline \multirow{2}{*}{ Adverse Event } & \multicolumn{3}{c}{ Number $(\%)$ of Subjects } \\
\cline { 2 - 4 } & Placebo $(N=47)$ & Nemiralisib $1000 \mu \mathrm{g}(N=48)$ & Total $(N=50)$ \\
\hline Total & $15(32)$ & $25(52)$ & $31(62)$ \\
Cough & $4(9)$ & $17(35)$ & $19(38)$ \\
Nasopharyngitis & $5(11)$ & $1(2)$ & $6(12)$ \\
Headache & $3(6)$ & $1(2)$ & $4(8)$ \\
Throat irritation & 0 & $2(4)$ & $2(4)$ \\
Oropharyngeal pain & $1(2)$ & $1(2)$ & $2(4)$ \\
Rhinitis & $2(4)$ & 0 & $2(4)$ \\
Bone contusion & 0 & $1(2)$ & $1(2)$ \\
Diarrhea & 0 & $1(2)$ & $1(2)$ \\
Dysphonia & 0 & $1(2)$ & $1(2)$ \\
Dyspnoea exertional & 0 & $1(2)$ & $1(2)$ \\
Fall & 0 & $1(2)$ & $1(2)$ \\
Hand fracture & 0 & $1(2)$ & $1(2)$ \\
Hypersensitivity & 0 & $1(2)$ & $1(2)$ \\
Influenza & 0 & $1(2)$ & $1(2)$ \\
Migraine & 0 & $1(2)$ & $1(2)$ \\
Muscle strain & 0 & $1(2)$ & $1(2)$ \\
Oral herpes & 0 & $1(2)$ & $1(2)$ \\
Rash & 0 & $1(2)$ & $1(2)$ \\
Sciatica & $1(2)$ & $1(2)$ \\
Sputum increased & 0 & $1(2)$ & $1(2)$ \\
Thermal burn & 0 & $1(2)$ & $1(2)$ \\
Arthralgia & 0 & 0 & $1(2)$ \\
Back pain & $1(2)$ & 0 & $1(2)$ \\
Dizziness & $1(2)$ & 0 & $1(2)$ \\
Dyspnoea & $1(2)$ & 0 & $1(2)$ \\
Epicondylitis & $1(2)$ & 0 & $1(2)$ \\
Migraine with aura & $1(2)$ & 0 & $1(2)$ \\
Respiratory tract infection & $1(2)$ & 0 & \\
\hline & $1(2)$ & & \\
\hline
\end{tabular}


TABLE 5

Summary of statistical analysis of inflammatory cytokine biomarkers (PD population)

\begin{tabular}{|c|c|c|c|c|c|c|c|}
\hline \multirow{2}{*}{ Cytokine (pg/ml) } & \multicolumn{2}{|r|}{ Placebo $(N=16)$} & \multicolumn{2}{|c|}{ Nemiralisib $1000 \mu \mathrm{g}(N=16)$} & \multirow[b]{2}{*}{$\begin{array}{l}\text { Posterior Median } \\
\text { Nemiralisib/Placebo (95\% CrI) }\end{array}$} & \multirow[b]{2}{*}{$\begin{array}{c}\text { Nemiralisib/Placebo } \\
\% \text { Inhibition }\end{array}$} & \multirow[b]{2}{*}{$\begin{array}{l}\text { Posterior Probability } \\
\text { of True Ratio }>0 \%\end{array}$} \\
\hline & $n$ & $\begin{array}{l}\text { Posterior Median Change } \\
\text { from Baseline ( } 95 \% \text { CrI })\end{array}$ & $n$ & $\begin{array}{l}\text { Posterior Median Change } \\
\text { from Baseline ( } 95 \% \text { CrI })\end{array}$ & & & \\
\hline IL-5 & $15^{a}$ & $1.06(0.65,1.76)$ & $15^{a}$ & $0.88(0.54,1.41)$ & $0.83(0.48,1.42)$ & $17 \%$ & $78 \%$ \\
\hline IL-8 & 15 & $1.01(0.54,1.91)$ & 16 & $0.94(0.51,1.73)$ & $0.92(0.54,1.58)$ & $8 \%$ & $63 \%$ \\
\hline IL-13 & 16 & $1.27(0.90,1.82)$ & 16 & $1.18(0.82,1.68)$ & $0.93(0.61,1.40)$ & $7 \%$ & $64 \%$ \\
\hline TNF- $\alpha$ & 16 & $0.95(0.57,1.58)$ & 16 & $0.99(0.60,1.67)$ & $1.04(0.62,1.75)$ & $-4 \%$ & $44 \%$ \\
\hline
\end{tabular}

${ }^{a}$ Seven of 15 values for IL-5 were imputed as half the lower limit of quantification.

Collectively, these data suggest that treatment with nemiralisib may reduce proinflammatory cytokine levels in the lungs of these patients.

\section{Discussion}

There remains an unmet need for novel therapies for patients with uncontrolled asthma. Nemiralisib, a potent and highly selective PI3K $\delta$ inhibitor, has previously been studied in patients with stable COPD and in patients experiencing a COPD exacerbation (Cahn et al., 2017; https://www.clinicaltrials.gov/ ct2/show/NCT02294734). The present study was the first time that nemiralisib was administered via the inhaled route to patients with persistent, uncontrolled asthma, treated only with an intermittent short-acting $\beta_{2}$-agonist or other noncorticosteroid controllers. The study was undertaken using the same dose shown to be effective, and with an acceptable safety profile, in patients with COPD. Our aim was to investigate the efficacy, safety, tolerability, and PK of nemiralisib in patients with asthma. The results demonstrated no discernible difference in trough $\mathrm{FEV}_{1}$ from baseline between nemiralisib and placebo treatment at day 28 (primary endpoint). These observations were also supported by most secondary endpoints. At day 14, sputum IL-5, IL-13, IL-6, and IL-8 levels were reduced by a median of $17 \%, 7 \%, 15 \%$, and $8 \%$, respectively, when comparing nemiralisib with placebo. Although the effects on proinflammatory cytokines suggest that nemiralisib was able to inhibit PI3K $\delta$ in the lung, this did not translate into a meaningful clinical improvement in this group of patients.

When the primary endpoint of a study is not achieved, clarity on whether drug plasma levels were sufficient is crucial for a correct interpretation of the data. The PK data demonstrated that nemiralisib was quickly absorbed into the systemic circulation and that the steady state level was attained after a week of treatment, which was consistent with the previous COPD study with nemiralisib (Cahn et al., 2017). Notably, similar plasma concentrations of nemiralisib were observed in patients with asthma and patients with stable COPD; mean trough concentrations ranged from 521 to $649 \mathrm{pg} / \mathrm{ml}$ in patients with asthma and from 604 to $711 \mathrm{pg} / \mathrm{ml}$ in those with stable COPD. Mean peak concentrations of nemiralisib at 5-10 minutes postdose were 1189 and $1109 \mathrm{pg} / \mathrm{ml}$, in patients with asthma and COPD, respectively (Cahn et al., 2017).

The presence of proinflammatory cytokines in induced sputum derived from patients with persistent, uncontrolled asthma aligns with the role of PI3K $\delta$ in promoting cytokine production and airway inflammation (Way et al., 2016). Although the levels of inhibition observed were modest, treatment with nemiralisib reduced the levels of sputumderived proinflammatory cytokines (including IL-5, IL-13, IL-6, and IL-8), relative to placebo. Reductions in IL-6 and IL-8 (although in some cases nonsignificant) have been observed in broadly similar populations treated with ICS (Inoue et al., 1999; Carpagnano et al., 2005; Zuiker et al., 2015); improvements in lung function parameters were also observed in these studies. Furthermore, reductions in IL-6 and IL-8 sputum levels were observed following 14-day nemiralisib inhalation in patients with stable COPD (reductions of $29 \%$ and $32 \%$ in IL-6 and IL-8, respectively) (Cahn et al., 2017). More recently, a study using the same dose of nemiralisib $(1000 \mu \mathrm{g})$ administered to patients with COPD experiencing exacerbations achieved its primary efficacy endpoint (https:// www.clinicaltrials.gov/ct2/show/NCT02294734).

Similar to our study, a randomized, double-blind, placebo-controlled, crossover study of another PI3K inhibitor, duvelisib, which inhibits both $\mathrm{PI} 3 \mathrm{~K} \delta$ and $\mathrm{PI} 3 \mathrm{~K} \gamma$ and is given via the oral route, did not meet the primary efficacy assessment of the maximum decrease in $\mathrm{FEV}_{1}$, following an allergen challenge in patients with asthma (Schmalbach et al., 2015). Significant improvements were observed in the secondary endpoints of $\mathrm{FEV}_{1}$ area under the curve and in methacholine challenge, suggesting some activity of this compound in patients with mild asthma (Schmalbach et al., 2015). The difference between the current nemiralisib study and the study with duvelisib may be due to route of administration (inhaled vs. oral) and/or inhibition of one (PI3K $\delta$ ) versus two (PI3K $\delta$ and PI3K $\gamma$ ) PI3K pathways.

Activation of SHIP1 with AQX-1125 via the oral route in patients with mild-to-moderate asthma was shown to significantly attenuate allergen-induced responses, accompanied by a trend toward reduced recruitment of eosinophils, neutrophils, and macrophages (Leaker et al., 2014). Whether the route of administration or whether activation of SHIP1 results in a more effective downregulation of the $\mathrm{PI} 3 \mathrm{~K} \delta$ pathway remains to be elucidated.

Overall, nemiralisib was well-tolerated. The most commonly reported AE was short-duration post-inhalation cough, which was generally considered to be mild or moderate in intensity and did not lead to patients discontinuing treatment. The presence of a short-duration post-inhalation cough in some patients was also reported in the nemiralisib study in patients with stable COPD (Cahn et al., 2017).

Although there was an improvement in ACT score with nemiralisib, the score remained less than 20 at day 28 in both groups, indicating a lack of good asthma control in both groups. In addition, although the mean daily frequency of 
rescue medication was slightly lower in the nemiralisib group compared with placebo, the percentage of rescue-free days was also lower in the nemiralisib group, again indicating a lack of good control with nemiralisib alone.

FeNO can help identify airway inflammation and can be used as a predictor of ICS response (http://www.niox.com/ Global/Documents/ATS-recommendations-for-interpretingFeNO.pdf). In the current study, a small improvement in FeNO was observed following nemiralisib versus placebo at the start of the treatment period (days 7 and 14), but the difference was negligible at day 28 , suggesting the effect was not sustained over time.

The absence of a meaningful clinical improvement in this group of patients with asthma treated with nemiralisib may also be explained by low levels of inflammation in these patients. The geometric mean baseline FeNO of 28.64 parts per billion suggests that the patient population had generally mild asthma. Furthermore, whereas the PD findings suggest that nemiralisib is able to inhibit the production of inflammatory cytokines in the lungs, thus potentially reducing inflammation, there was no clinically relevant change in FeNO at the end of the treatment period, suggesting little change to the extent of eosinophilic airway inflammation in these patients following nemiralisib inhalation.

The precision of treatment comparisons in this study was improved by using a crossover design compared with a parallel group design, because the comparisons would be made within an individual rather than between individuals. A washout period of 4 weeks was considered sufficient based on the steady state and PD effects of previous studies with nemiralisib. Although the sample size was small, it was sufficient to detect meaningful changes in $\mathrm{FEV}_{1}$ (the primary endpoint); however, the study was unable to measure changes in the rate of exacerbations and patient-reported outcomes, both of which would require a larger sample size and/or longer study duration. Hence, larger studies with a longer period of treatment are required to further investigate the effect of nemiralisib on these endpoints in patients with asthma. In addition, an alternative endpoint could have been to explore the effect of nemiralisib on methacholine-evoked bronchial hyper-reactivity; however, this would have been an additional procedure for the patients to undertake, and it is not widely recognized as a proof-of-concept efficacy endpoint.

In conclusion, this study has shown that nemiralisib demonstrates an acceptable safety and tolerability profile in patients with persistent, uncontrolled asthma, but not currently on ICS treatment, with the most common AE a shortduration post-inhalation cough. Inhalation of nemiralisib appears to act upon the PI3K $\delta$ pathway, leading to a reduction in sputum proinflammatory cytokine levels, including IL-5, IL-6, IL-8, and IL-13 when compared with placebo, suggestive of reduced airway inflammation in these patients; however, this effect did not translate to marked clinical improvements in lung function in this population. Based on the collective results of our clinical studies in both patients with COPD and asthma, and the published observations in patients with the activating mutation in $\mathrm{PI} 3 \mathrm{~K} \delta$ (APDS) who suffer from severe recurrent respiratory infections, we hypothesize that nemiralisib may be most effective in patients who are colonized with bacteria and frequently exacerbate, regardless whether they have asthma or COPD. Further studies are needed to investigate the potential efficacy of nemiralisib in patients with asthma with other specific more severe phenotypes, including those who are colonized with bacteria and frequently exacerbate.

\section{Acknowledgments}

Medical writing support was provided by Leigh O'Connor of Fishawack Indicia, UK, and was funded by GSK.

\section{Authorship Contributions}

Participated in research design: Khindri, Begg, Montembault, Cui, Robertson, Hamblin, Hessel.

Contributed new reagents or analytic tools: Hogg, Wajdner, Ludwig-Sengpiel, Kornmann.

Performed data analysis: Khindri, Cahn, Begg, Leemereise, Cui, Wajdner, Yang, Robertson, Hamblin, Hessel.

Wrote or contributed to the writing of the manuscript: Khindri, Cahn, Begg, Montembault, Leemereise, Cui, Hogg, Wajdner, Yang, Robertson, Hamblin, Ludwig-Sengpiel, Kornmann, Hessel.

\section{References}

Ali K, Bilancio A, Thomas M, Pearce W, Gilfillan AM, Tkaczyk C, Kuehn N, Gray A, Giddings J, Peskett E, et al. (2004) Essential role for the p110delta phosphoinositide 3-kinase in the allergic response. Nature 431:1007-1011.

American Thoracic Society; European Respiratory Society (2005) ATS/ERS recommendations for standardized procedures for the online and offline measurement of exhaled lower respiratory nitric oxide and nasal nitric oxide, 2005. Am J Respir Crit Care Med 171:912-930.

Cahn A, Hamblin JN, Begg M, Wilson R, Dunsire L, Sriskantharajah S, Montembault M, Leemereise CN, Galinanes-Garcia L, Watz H, et al. (2017) Safety, pharmacokinetics and dose-response characteristics of GSK2269557, an inhaled PI3K $\delta$ inhibitor under development for the treatment of COPD. Pulm Pharmacol Ther 46:69-77.

Carpagnano GE, Foschino Barbaro MP, Resta O, Gramiccioni E, Valerio NV, Bracciale P, and Valerio G (2005) Exhaled markers in the monitoring of airways inflammation and its response to steroid's treatment in mild persistent asthma. Eur J Pharmacol 519:175-181.

Chantry D, Vojtek A, Kashishian A, Holtzman DA, Wood C, Gray PW, Cooper JA and Hoekstra MF (1997) p110delta, a novel phosphatidylinositol 3-kinase catalytic subunit that associates with p85 and is expressed predominantly in leukocytes. $J$ Biol Chem 272:19236-19241.

Clayton E, Bardi G, Bell SE, Chantry D, Downes CP, Gray A, Humphries LA, Rawlings D, Reynolds H, Vigorito E, et al. (2002) A crucial role for the p110 subunit of phosphatidylinositol 3-kinase in B cell development and activation. $J$ Exp Med 196:753-763.

Deo SS, Mistry KJ, Kakade AM, and Niphadkar PV (2010) Role played by Th2 type cytokines in IgE mediated allergy and asthma. Lung India 27:66-71.

Down K, Amour A, Baldwin IR, Cooper AW, Deakin AM, Felton LM, Guntrip SB, Hardy C, Harrison ZA, Jones KL, et al. (2015) Optimization of novel indazoles as highly potent and selective inhibitors of phosphoinositide 3-kinase $\delta$ for the treatment of respiratory disease. J Med Chem 58:7381-7399.

Dweik RA, Boggs PB, Erzurum SC, Irvin CG, Leigh MW, Lundberg JO, Olin AC Plummer AL, and Taylor DR; American Thoracic Society Committee on Interpretation of Exhaled Nitric Oxide Levels (FENO) for Clinical Applications (2011) An official ATS clinical practice guideline: interpretation of exhaled nitric oxide levels (FENO) for clinical applications. Am J Respir Crit Care Med 184: $602-615$.

Franciosi LG, Page CP, Celli BR, Cazzola M, Walker MJ, Danhof M, Rabe KF, and Della Pasqua OE (2006) Markers of disease severity in chronic obstructive pulmonary disease. Pulm Pharmacol Ther 19:189-199.

Hacievliyagil SS, Gunen H, Mutlu LC, Karabulut AB, and Temel I (2006) Association between cytokines in induced sputum and severity of chronic obstructive pulmonary disease. Respir Med 100:846-854.

Inoue $\mathrm{H}$, Aizawa $\mathrm{H}$, Fukuyama $\mathrm{S}$, Takata $\mathrm{S}$, Matsumoto $\mathrm{K}$, Shigyo M, Koto $\mathrm{H}$, and Hara N (1999) Effect of inhaled glucocorticoid on the cellular profile and cytokine levels in induced sputum from asthmatic patients. Hai 177:53-62.

Leaker BR, Barnes PJ, O'Connor BJ, Ali FY, Tam P, Neville J, Mackenzie LF, and MacRury T (2014) The effects of the novel SHIP1 activator AQX-1125 on allergen-induced responses in mild-to-moderate asthma. Clin Exp Allergy 44: 1146-1153.

Lee KS, Lee HK, Hayflick JS, Lee YC, and Puri KD (2006) Inhibition of phosphoinositide 3-kinase delta attenuates allergic airway inflammation and hyperresponsiveness in murine asthma model. FASEB $J$ 20:455-465.

Lipworth BJ (1999) Systemic adverse effects of inhaled corticosteroid therapy: a systematic review and meta-analysis. Arch Intern Med 159:941-955.

Maneechotesuwan K, Essilfie-Quaye S, Kharitonov SA, Adcock IM, and Barnes PJ (2007) Loss of control of asthma following inhaled corticosteroid withdrawal is associated with increased sputum interleukin-8 and neutrophils. Chest 132 : 98-105.

Matangkasombut P, Pichavant M, Dekruyff RH, and Umetsu DT (2009) Natural killer T cells and the regulation of asthma. Mucosal Immunol 2:383-392.

Menzella F, Lusuardi M, Montanari G, Galeone C, Facciolongo N, and Zucchi L (2016) Clinical usefulness of mepolizumab in severe eosinophilic asthma. Ther Clin Risk Manag 12:907-916. 
Miller MR, Hankinson J, Brusasco V, Burgos F, Casaburi R, Coates A, Crapo R, Enright P, van der Grinten CP, Gustafsson P, et al.; ATS/ERS Task Force (2005) Standardisation of spirometry. Eur Respir $J$ 26:319-338.

Nashed BF, Zhang T, Al-Alwan M, Srinivasan G, Halayko AJ, Okkenhaug K, Vanhaesebroeck B, Hayglass KT, and Marshall AJ (2007) Role of the phosphoinositide 3-kinase p110delta in generation of type 2 cytokine responses and allergic airway inflammation. Eur J Immunol 37:416-424.

Norzila MZ, Fakes K, Henry RL, Simpson J, and Gibson PG (2000) Interleukin8 secretion and neutrophil recruitment accompanies induced sputum eosinophil activation in children with acute asthma. Am J Respir Crit Care Med 161:769-774.

Okkenhaug K, Ali K, and Vanhaesebroeck B (2007) Antigen receptor signalling: a distinctive role for the p110delta isoform of PI3K. Trends Immunol 28:80-87.

Okkenhaug K, Bilancio A, Farjot G, Priddle H, Sancho S, Peskett E, Pearce W, Meek SE, Salpekar A, Waterfield MD, et al. (2002) Impaired B and T cell antigen receptor signaling in p110delta PI 3-kinase mutant mice. Science 297:1031-1034.

Park SJ, Min KH, and Lee YC (2008) Phosphoinositide 3-kinase $\delta$ inhibitor as a novel therapeutic agent in asthma. Respirology 13:764-771.

Patton DT, Garden OA, Pearce WP, Clough LE, Monk CR, Leung E, Rowan WC Sancho S, Walker LS, Vanhaesebroeck B, et al. (2006) Cutting edge: the phosphoinositide 3-kinase p110 delta is critical for the function of CD4+CD25+Foxp3+ regulatory T cells. J Immunol 177:6598-6602.

Reddy D and Little FF (2013) Glucocorticoid-resistant asthma: more than meets the eye. J Asthma 50:1036-1044.

Robinson D, Hamid Q, Bentley A, Ying S, Kay AB, and Durham SR (1993) Activation of CD4+ T cells, increased TH2-type cytokine mRNA expression, and eosinophil recruitment in bronchoalveolar lavage after allergen inhalation challenge in patients with atopic asthma. J Allergy Clin Immunol 92:313-324.

Robinson DS, Hamid Q, Ying S, Tsicopoulos A, Barkans J, Bentley AM, Corrigan C Durham SR, and Kay AB (1992) Predominant TH2-like bronchoalveolar T-lymphocyte population in atopic asthma. N Engl J Med 326:298-304
Schmalbach T, Fuhr R, Albayaty M, Allen K, Douglas M, Dunbar J, McLaughlin J, Alexander L, and McKee C (2015) Duvelisib, a PI3K- $\delta, \gamma$ inhibitor, in subjects with mild asthma. European Respiratory Journal 46:PA2122.

Soriano JB, Abajobir AA, Abate KH, Abera SF, Agrawal A, Ahmed MB, Aichour AN, Aichour I, Aichour MTE, Alam K, et al.; GBD 2015 Chronic Respiratory Disease Collaborators (2017) Global, regional, and national deaths, prevalence, disabilityadjusted life years, and years lived with disability for chronic obstructive pulmonary disease and asthma, 1990-2015: a systematic analysis for the Global Burden of Disease Study 2015. Lancet Respir Med 5:691-706.

Stark A-K, Sriskantharajah S, Hessel EM, and Okkenhaug K (2015) PI3K inhibitors in inflammation, autoimmunity and cancer. Curr Opin Pharmacol 23:82-91.

Vanhaesebroeck B, Guillermet-Guibert J, Graupera M, and Bilanges B (2010) The emerging mechanisms of isoform-specific PI3K signalling. Nat Rev Mol Cell Biol 11:329-341.

Way EE, Trevejo-Nunez G, Kane LP, Steiner BH, Puri KD, Kolls JK, and Chen K (2016) Dose-dependent suppression of cytokine production from $\mathrm{T}$ cells by a novel phosphoinositide 3-kinase delta inhibitor. Sci Rep 6:30384.

Wu AC, Paltiel AD, Kuntz KM, Weiss ST, and Fuhlbrigge AL (2007) Costeffectiveness of omalizumab in adults with severe asthma: results from the Asthma Policy Model. J Allergy Clin Immunol 120:1146-1152.

Yokoyama A, Kohno N, Fujino S, Hamada H, Inoue Y, Fujioka S, Ishida S, and Hiwada K (1995) Circulating interleukin-6 levels in patients with bronchial asthma. Am J Respir Crit Care Med 151:1354-1358.

Zuiker RG, Ruddy MK, Morelli N, Mogg R, Rivas VM, van Dyck K, De Lepeleire I, Tanen MR, Boot JD, Kamerling IM, et al. (2015) Kinetics of TH2 biomarkers in sputum of asthmatics following inhaled allergen. European Clinical Respiratory Journal 2:28319.

Address correspondence to: Dr. Edith M. Hessel, Refractory Respiratory Inflammation Discovery Performance Unit, GSK, Gunnels Wood Road, Stevenage SG1 2NY, United Kingdom. E-mail: edith.m.hessel@gsk.com 